\title{
Red Ecoturística de Íntag: experiencia de una organización y gestión de economía solidaria
}

\author{
Íntag Ecotourism Network: a solidarity economy \\ organization and management experience
}

Rede Eco turística de Íntag: experiência de uma organização e gestão de economia solidária

\author{
Ana Cristina Beltrán \\ Instituto de Altos Estudios Nacionales (Quito, Ecuador) \\ acrisbel55@gmail.com
}

DOI: https://doi.org/10.32719/25506641.2019.6.3

Fecha de presentación: 1 de junio de 2019 • Fecha de aceptación: 12 de agosto de 2019 Artículo de investigación

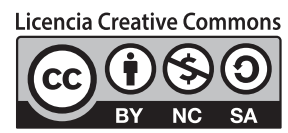




\section{Resumen}

El presente artículo sistematiza el trabajo desarrollado por las redes de economía solidaria. El objetivo central es analizar los procesos de funcionamiento de la Red Ecoturística de Íntag, sus relaciones políticas, socioeconómicas y culturales. Es una investigación cualitativa, método etnográfico, donde se buscó evidenciar el funcionamiento, gestión, organización de la red analizada y su contribución al desarrollo territorial. Los principales hallazgos indican que la red obedece a un trabajo comunitario centrado en el turismo ecológico del sector, pero que se refuerza con otras actividades económicas, como la alimentación y hospedaje, y que, en algunos casos, estas se han desarrollado bajo el enfoque de género, lo que apoya el desarrollo socioeconómico de las mujeres participantes. No obstante, la minería a cielo abierto y los proyectos hidroeléctricos a gran escala en el área de influencia, se han constituido como una amenaza real al crecimiento de la red, pues estas actividades tienen consecuencias sobre el turismo y actividades complementarias.

Palabras clave: Redes de colaboración solidaria, desarrollo integral local, economía solidaria. JEL: A13 Relación de la economía con los valores sociales.

\section{Abstract}

This article systematizes the work developed by solidarity economy networks. The main objective is to analyze the operating processes of Entagistics Network of Íntag, its political, socio-economic and cultural relations. It is a qualitative research, ethnographic method, where it was sought to demonstrate the operation, management, organization of the analyzed network and its contribution to territorial development. The main findings indicate that the Network is due to community work focused on ecological tourism in the sector, but it is reinforced by other economic activities, such as food and lodging, and that, in some cases, these have been developed under the gender approach, which supports the socio-economic development of the participating women. However, open pit mining and large-scale hydroelectric projects in the area of influence have been constituted as a real threat to the Network growth since these activities have consequences on tourism and complementary activities.

Keywords: Solidarity collaboration networks, local integral development, solidarity economy. JEL: A13 Relationship of the economy with social values.

\section{Resumo}

O presente artículo sistematiza o trabalho desenvolvido pelas redes de economia solidária. O objetivo central é analisar os processos de funcionamento da Rede Eco turística de Íntag, suas relações políticas, socioeconômicas e culturais. É uma investigação qualitativa, método etnográfico, onde se buscou evidenciar o funcionamento, gestão, organização da rede analisada e sua contribuição ao desenvolvimento territorial. As principais descobertas indicam que, a Rede, obedece a um trabalho comunitário centrado no turismo ecológico do setor, mas que se reforça com outras atividades econômicas, como a alimentação e hospedagem, e que, em alguns casos, estas foram desenvolvidas sob o 
enfoque de gênero, o que apoia o desenvolvimento socioeconômico das mulheres participantes. Não obstante, a mineração a céu aberto e os projetos hidroelétricos em grande escala na área de influência, se constituíram como uma ameaça real ao crescimento da Rede, pois estas atividades têm consequências sobre o turismo e atividades complementárias.

Palavras-chave: Redes de colaboração solidária, desenvolvimento integral local, economia solidária.

JEL: A13 Relação da economia com os valores sociais.

\section{Introducción}

E ste trabajo pretende resaltar una forma de organización y gestión solidaria llamada redes de colaboración solidaria, la cual permite un desarrollo territorial desde las necesidades de los actores, con el fin de mejorar las condiciones y la calidad de vida de sus miembros y la comunidad.

Para entender el contexto solidario de las actividades desarrolladas por las redes de colaboración solidaria, se realizó un análisis de las experiencias presentes en América Latina y Ecuador, tales como la Red de Mujeres Solidarias y Redes de Trueque en Argentina, la Central Cooperativa de Servicios Sociales en Venezuela, la Red de Recicladores en Colombia, y el estudio de la Red de Canastas Comunitarias en Ecuador.

La investigación bibliográfica condujo a determinar que no existen muchas investigaciones sobre redes de colaboración solidaria en Ecuador, y por ello se tomó como unidad de observación a una de ellas: la Red Ecoturística de Íntag (REI), ubicada en la provincia de Imbabura, con la cual se realizó un estudio a profundidad y se conoció su funcionamiento bajo el enfoque de una economía alternativa.

El análisis de redes de colaboración solidaria es un aporte importante a la construcción de una nueva sociedad que actúa bajo otra lógica distinta, donde prima el bienestar del ser humano sobre la acumulación desmedida del capital. En la zona de Íntag se mira cómo se desenvuelve y cómo se sobrepasa el ámbito económico hacia una visión social, política y cultural en todas las actividades, creando lazos que se retroalimentan entre sí, en beneficio de todos sus miembros y su comunidad en territorio, a través de la actividad turística responsable y sostenible con el medioambiente. 
El objetivo general del presente estudio es analizar los procesos de funcionamiento de la Red Ecoturística de Íntag, sus relaciones políticas, sociales, económicas, culturales, y los lazos que le han permitido conformarse como red, para determinar su vinculación con los preceptos de la economía solidaria, y cómo esta sinergia colectiva les ha permitido fortalecer los fundamentos y las dinámicas en las que una red de economía solidaria se origina y desarrolla.

La información generada es entendida e interpretada a través del concepto de "red de colaboración solidaria" propuesto por Mance (2010), que está enmarcada en la Teoría de la Economía Solidaria, donde se unen las reflexiones sobre los principios solidarios de Coraggio (2013), las fases del proceso económico solidario que menciona Jácome (2014) y se complementan con la definición de desarrollo territorial de Gallicchio (2004).

La información fue obtenida a través de la investigación cualitativa, cuyo interés se basa en la comprensión de la problemática mediante las voces de los actores y el acercamiento directo a su realidad del sector, enfatizando en la aplicación del método etnográfico y la aplicación de entrevistas semiestructuradas, para lograr así entender su contribución al desarrollo territorial.

Con esta investigación se pretende visibilizar un caso de red de colaboración solidaria en Ecuador, que incentive a esta nueva forma de trabajo conjunto, a la elaboración de nuevos trabajos teóricos, y que proporcione información relevante para el diseño de políticas públicas de redes de colaboración solidaria que, además, generen una conciencia ecológica y de género.

Además del capítulo introductorio, el presente artículo contiene las siguientes secciones adicionales donde se desarrolla el marco teórico; la metodología, donde se realiza un breve análisis de las redes de colaboración solidaria dentro de un enfoque más amplio que es la economía solidaria; la presentación de resultados, donde se presenta el contexto del estudio de caso analizado, los métodos utilizados y los principales resultados obtenidos; y las discusiones finales. 


\section{Marco teórico}

Para entender esta experiencia desde el enfoque solidario, se interpretó a través de los conceptos de economía solidaria, de las redes de colaboración solidaria, principios solidarios, fases del proceso económico dentro del sector de economía popular y solidaria y desarrollo integral local, siendo estas alternativas al desarrollo territorial.

Conceptualmente, se parte de una de las teorías del posdesarrollo como una economía alternativa: la economía solidaria y la de colaboración solidaria. El término de economía solidaria surge en la década de 1990 por productores y consumidores en organizaciones de diversas formas como cooperativas, comedores, redes, nudos de trueque basadas en principios de cooperación, autonomía y gestión democrática, en el cual su objetivo es el bien común sobre el interés individual, la ganancia material es para todos, se sociabilizan los recursos productivos y se establecen criterios de equidad. Como lo mencionan Laville y Gaiger (2013), la solidaridad se promueve entre sus miembros y hacia los más vulnerables en la sociedad a través del vínculo social de reciprocidad y la participación ciudadana, así se pueden cubrir varias áreas de salud, educación y de conservación ambiental.

Desde la perspectiva de la economía solidaria, se deben repensar las relaciones económicas más allá de la búsqueda del máximo beneficio, sobre la base de una relación de producción, distribución, consumo y financiación, fundamentados en la justicia, cooperación, reciprocidad y ayuda mutua, donde las personas y su trabajo se sitúen en el centro del sistema. Por ello, Mance (2011) define como redes de colaboración solidaria a las manifestaciones con lazos económicos, políticos, sociales y culturales que se retroalimentan entre sí, haciendo posible la construcción de otro tipo de sociedad distinta dentro de un sistema económico alternativo al capitalismo; además, le da un peso importante a la democracia participativa que enlaza lo administrativo con lo político dentro de la economía solidaria.

Así mismo, Mance $(2013,297)$ menciona que "Las características básicas de las redes de colaboración solidaria son la autopoiesis, intensividad, extensividad, diversidad, integralidad, retroalimentación de flujo de valor, flujo de información, flujo de materias y agregación, interconectándose en 
relaciones económicas, políticas, sociales y culturales", de tal forma que la gestión de redes se puede analizar de acuerdo con ciertos parámetros endógenos y exógenos de las mismas, como son: participantes, iniciativa, alcance, modelo de gestión, grados de formalización, grados de articulación institucional, problemas que se intenta resolver (Caracciolo Basco y Foti Laxalde 2003).

Al interpretar estas conceptualizaciones en vida comunal en las redes, se puede notar que ya tienen una visión integral avanzada, debido a que los actores miran como natural el trabajo en conjunto, que beneficie siempre a todos los miembros de la comunidad, respetando al ecosistema en todas sus actividades, tal como lo menciona Roux $(2007,7)$ :

El escenario alternativo basado en el verdadero Sumak Kawsay, sea donde se cumple el sueño de las comunidades de convivencia armónica con la Pachamama, que cuidan y dependen de su biodiversidad, favorecen la posibilidad de alcanzar la felicidad en un ambiente sano, con equidad, seguridad alimentaria, a través de la ejecución de proyectos sustentables, que pueden ser proyectos de agroecología y de turismo comunitario, cuando este toma como eje la protección ambiental y defensa de los territorios ancestrales.

Complementando lo anterior, estos términos, de acuerdo con Jubeto, Guridi y Fernández-Villa (2014), toman como base los principios de la economía comunitaria andina, en relación al papel de los seres humanos en el planeta y prácticas de reciprocidad y el trabajo comunitario, el medioambiente y el desarrollo sustentable sobre otros intereses, lo cual implica en la forma de vivir para alcanzar el desarrollo del ser humano de una manera integral (39). De tal forma, se pretende incorporar a la gestión de la actividad económica los valores universales que deben regir la sociedad y las relaciones entre toda la ciudadanía: equidad, justicia, fraternidad económica, solidaridad social y democracia directa, generando una nueva forma de producir, de consumir y de distribuir, como una alternativa viable y sostenible para la satisfacción de las necesidades individuales y globales, aspirando a consolidarse como un instrumento de transformación social.

Estas conceptualizaciones se ven implícitas en las actividades diarias que realizan los actores de una red de colaboración solidaria dentro de las fases del proceso económico solidario, las mismas que son: producción solidaria, comercialización justa y solidaria, consumo responsable y solidario y finanzas solidarias. Este proceso de interrelación, como lo menciona Muñoz 
(citado por Jácome 2014), también implica que las prácticas de economía popular y solidaria se relacionen con el Estado y gobiernos locales, organismos internacionales y regionales, la cooperación internacional y el mercado.

Además, es importante destacar que al ser las redes una forma de organización en el sector de economía popular y solidaria deben desarrollar los cuatro principios básicos de la economía de Polanyi (citado en Jácome 2014) como son la reciprocidad (dar, recibir y devolver), autarquía (resolver las necesidades de sus miembros), redistribución (entregar a alguien los excedentes para ser distribuidos como ellos crean conveniente) y la relación con el mercado (que no sea únicamente una relación mercantilista), con el fin de desarrollarse bajo un objeto social que las una a través de una actividad económica sostenible, característica de cualquier forma organizativa de este sector solidario.

Adicionalmente, el desarrollo integral local se lo entiende como una nueva forma de mirar y de actuar desde el territorio, en donde existe mayor participación de los actores y un mejor uso de los recursos. Permite un trabajo desde el interior hacia el exterior. Gallicchio (2004) menciona que este tipo de descentralización favorece a que en los procesos existan contenidos de cambio social y así visibilizar al territorio, generando sujetos en democracia que propicien una relación consciente entre el Estado, mercado y actores.

\section{Datos generales de Íntag}

Íntag se encuentra en una zona subtropical de aproximadamente 1.600 $\mathrm{km}^{2}$; ubicada en el piedemonte occidental de la cordillera de los Andes, al occidente de la provincia de Imbabura, al norte de Ecuador. Su altura oscila entre 575 a $3.800 \mathrm{msnm}$, su temperatura promedio anual es de $18^{\circ} \mathrm{C}$ y la precipitación promedio es de $1.500 \mathrm{~mm}$. Esta zona de transición entre los páramos y el subtrópico genera una serie de microclimas que propician una rica biodiversidad y variada producción agropecuaria.

El territorio está conformado por siete parroquias rurales: García Moreno, Apuela, Cuellaje, Peñaherrera, Vacas Galindo y Plaza Gutiérrez, pertenecientes al cantón Cotacachi, y Selva Alegre, del cantón Otavalo. La diversa procedencia de los habitantes ha otorgado a esta zona una peculiar diversidad sociocultural, que incluye al pueblo mestizo de diversa procedencia andina, 
al pueblo afroecuatoriano originario del valle de Chota y al pueblo Otavalo (Roux 2013).

Las actividades económicas, en su mayoría, de acuerdo con datos del INEC (2010) son la agricultura y la manufactura de artesanías y productos cosméticos, que se basan en la elaboración de artículos a base de lufa, cabuya y tagua. Adicionalmente, en la última década se han fomentado actividades en ecoturismo, no obstante, esta ha sido mermada por los efectos negativos de la actividad minera.

La actividad minera en la zona de Íntag tiene más de 20 años de existencia, y se ha desarrollado entre la oposición de los habitantes del sector, quienes lograron que en 1997 el cantón Cotacachi, del cual Íntag es parte en su mayoría, fuera declarada región ecológica. En el año 2000 se creó una ley local que estableció la primera región ecológica en América Latina, pero a pesar de ello, en 2012 los gobiernos de Chile y Ecuador firmaron un contrato para reabrir un proyecto minero en Íntag.

La resistencia hacia la actividad minera, como lo mencionan Ortiz y Mena (2005), se da por el impacto socioeconómico y ambiental severo, generando problemas como la reubicación de familias, la proliferación de la delincuencia y la prostitución, entre otros. Así mismo, la concentración de metales tóxicos, tales como el plomo, ha causado daños a la salud, contaminación de ríos y quebradas, con niveles hasta cien veces más altos de los encontrados naturalmente en los ríos, y deforestación que afectó el hábitat de docenas de especies de aves, mamíferos y reptiles.

A finales de 2006 se crea la Red Ecoturística de Íntag, como iniciativa de los pobladores para agrupar, articular, organizar y regular las actividades turísticas, bajo una red de turismo, la misma que junto a once organizaciones $^{1}$ de diferentes parroquias y con el apoyo de la ONG española Fundación Ayuda en Acción, tuvieron como fin fortalecer institucionalmente a la red a nivel local y nacional, desarrollando un modelo de turismo comunitario propio, como eje transversal del desarrollo socioeconómico local, fomentando la generación de empleo, para evitar la migración y que sea una alternativa a las actividades extractivistas.

1. En esta investigación, para facilidad de comprensión se denominará organizaciones a todas estas asociaciones, consorcios, microempresas que integran la red debido a que muchas de ellas, por su actividad, deben registrarse donde la institución pública lo establezca. 
La REI actualmente es parte del Comité Intersectorial de Turismo de Cotacachi, perteneciente a la Asamblea de Unidad Cantonal, y en el ámbito nacional es miembro de la Federación Pluricultural de Turismo Comunitario del Ecuador (FEPTCE). El proyecto se propuso como objetivos fortalecer la infraestructura turística de la zona de Íntag, implementando instalaciones de interpretación turística-ambiental, con la creación de centros informáticos y de telecomunicaciones, la apertura y señalización de senderos y la creación de otros servicios anexos al turismo; además, promover el fortalecimiento de las capacidades locales e involucrar a los actores directamente vinculados a las actividades turísticas y a los que ofertan otro tipo de servicios.

De acuerdo con el Instituto Interamericano de Cooperación para la Agricultura (IICA 2013), el turismo en el territorio incluye variantes de actividades relacionadas con el esparcimiento: atractivos de aventura, turismo comunitario y ecoturismo. Este tipo de oferta turística se integró en la REI, una organización de segundo grado con personería jurídica otorgada por el Consejo de Desarrollo de las Nacionalidades y Pueblos de Ecuador (CODENPE). Así mismo, Íntag es una zona arqueológica que ha concitado el interés científico desde hace décadas, incluyendo el registro del lugar llamado Wariman (también conocido como Guallimán) en 1979.

\section{Metodología}

La presente investigación utilizó el método etnográfico; para el acercamiento a la experiencia de economía solidaria, se participó abiertamente con las personas de la red, organizaciones miembros, las instituciones públicas, privadas y personas de otras organizaciones de economía solidaria, a través de entrevistas semiestructuradas, observación de comportamientos, recabando información útil para la investigación, tal como lo proponen Hammersley y Atkinson (citado por Jácome 2014).

Con lo expuesto anteriormente se generó un conocimiento de los actores de la REI basada en los siguientes aspectos: i) observación de la REI; ii) interpretación teórica de lo que se ve en la experiencia; iii) preguntas concretas a los involucrados y no involucrados con la experiencia; iv) notas de campo; v) grabaciones de audio; y vi) formulación de conclusiones. 
El método etnográfico es útil, ya que permite analizar la experiencia con un contacto directo con la gente. Tal como lo menciona Jácome (2014), en este tipo de metodología el conocimiento se construye en el campo; con ello se pretende interpretar el significado de la red en su funcionamiento, gestión y prácticas que no se ven.

El trabajo etnográfico comenzó desde cero, porque no se tenía contacto alguno con la red. Primero, se buscó al informante clave que facilite el acercamiento a los miembros y a las organizaciones que la integran, que en este caso fue Franklin Vaca, presidente de la red en ese entonces; luego se presentaron cambios no esperados que generaron complicaciones para la investigación. Sin embargo, se consolidaron contactos en el proceso para asegurar los resultados cualitativos.

En relación a las entrevistas, estuvieron dirigidas a actores externos pertenecientes a instituciones públicas como: la Dirección de Turismo de Cotacachi, Instituto Nacional de Economía Popular y Solidaria de Ibarra y de Quito, y encargados del turismo comunitario en el Ministerio de Turismo. Así también, fue importante contar con el testimonio de organizaciones privadas relacionadas como el Centro Arqueológico Gualimán, hosterías de la zona, empresas de transportes, otras organizaciones de economía solidaria como Camari y la Casa de Íntag, y otros actores internos, con el fin de recoger las experiencias vividas por los miembros de las organizaciones, sus aspiraciones, sus anhelos en distintos escenarios en Íntag y su realidad frente a las actividades extractivistas como la minería, y la posición del turismo como eje estratégico del desarrollo local. A todo esto, se suma una investigación bibliográfica de casos similares y estadísticas proporcionadas por el Instituto Ecuatoriano de Estadística y Censos (INEC), relacionada con actividades económicas, nivel de instrucción y número de pobladores de los habitantes de Íntag.

Por último, el hecho de que la red estaba en proceso de reestructuración, durante la fase de investigación constituyó un obstáculo para obtener la información documentada interna e interpretar un histórico de la REI desde 2006, que junto a los conflictos ambientales impidió obtener datos específicos de la zona. No obstante, se recolectó la mayor cantidad de información en ese escenario, que permitió interpretar la parte social, política y cultural, y no únicamente lo económico. 


\section{Análisis de la Red Ecoturística de Íntag}

La red divide su funcionamiento en dos partes: administrativa y operativa. La parte administrativa está conformada por once organizaciones que son las participantes y se dedican a diferentes actividades: producción de café, fincas agroecológicas, manufactura de artesanías y de productos cosméticos, turismo, cabañas, albergues, actividades turísticas y deportes de aventura, con su principal atractivo turístico que son las Termas de Nangulvi; las organizaciones tienen un representante, cada una, para las reuniones de la asamblea general, y, además, un presidente, un secretario financiero, un secretario de actas y dos vocales que se eligen cada dos años. Para los cargos de apoyo operativos cuentan con una persona en el área de atención al cliente, un administrador, un coordinador local de ventas, un coordinador de proyectos y una persona que se dedica a la administración de la página web y otros medios publicitarios (F. V. ${ }^{2} 2016$, entrevista personal).

En cuanto a la parte operativa, la REI, en conjunto con las organizaciones, han desarrollado rutas turísticas en donde involucran los atractivos de la zona, y donde se ofrece alojamiento y alimentación, que se complementan con actividades como: talleres de artesanías, productos agrícolas y cosméticos en cada uno de los paquetes ofertados. Cabe resaltar que la coordinación de ventas de la red se encarga de atender a los clientes, a quienes se contacta por diferentes medios - como pueden ser una visita a las piscinas de Nangulvi (lugar donde se encuentran las oficinas de la red)-, o a través de canales publicitarios como la página web, agencias de turismo y personas recomendadas por otros canales, como es la coordinación de proyectos, donde la red trabaja con ONG que aportan al desarrollo de la zona (V. A. 2016, entrevista personal).

El estudio a profundidad de la Red Ecoturística de Íntag demuestra que, por su lógica de funcionamiento, principios y prácticas, es una organización de colaboración solidaria que ha contribuido con un objetivo social a través

2. Debido a un acuerdo de confidencialidad establecida entre el investigador y el participante acerca de cómo se manejará, administrará y difundirá la información privada de identificación, el presente artículo elaboró una estrategia que permite preservar la confidencialidad de las personas entrevistadas, el cual consiste en utilizar letras para identificar a los distintos involucrados. 
de la actividad económica, como se muestra en la figura 1, la cual fue creada con la información recopilada de las voces de los miembros de la red, miembros de las hosterías privadas, personas de la institución pública en base a la propia observación de la REI, interpretación teórica de la observación participante, notas de campo y grabación de entrevistas semiestructuradas.

De acuerdo con la figura 1, existe una dinámica vinculada con un trabajo conjunto comunitario, en donde predomina el bien común de todos sus miembros, pues involucran a cada una de las siete parroquias de Íntag, en donde se encuentran las organizaciones, generando un modelo de gestión propio de acuerdo con sus necesidades, que permita resolver los problemas de sus miembros y de la comunidad, logrando tener por el momento un alcance local. Pero no son un ente aislado, ellos trabajan de forma cooperativa con organizaciones privadas que están comprometidas con esta lucha por preservar su territorio a través de la colaboración solidaria, como es el sitio arqueológico Gualimán, el bosque de aves de la Cueva de los Tayos y algunas hosterías privadas. Además, se potencia esta sinergia colectiva, logrando comercializar los productos y servicios turísticos de cada una de las organizaciones de la red en las tiendas de comercio justo como son Camari y la Casa de Íntag, generando así más fuentes de empleo a través del turismo, e ingresos adicionales a las familias.

Adicionalmente, el trabajo que realizan las organizaciones de la REI, a través de proyectos, no tienen como objetivo generar ganancias, sino también capacitaciones que, en muchos casos, son brindadas por las instituciones públicas como el Ministerio de Turismo, el Instituto de Economía Popular y Solidaria (IEPS), juntas parroquiales y gobiernos locales (de los cuales no han recibido mayor apertura); apoyo de organismos internacionales, y de la Fundación PRODECI. Hay que destacar la labor del Consorcio Toaisan, conformado por pequeños líderes comunitarios, cuyo aporte a la REI ha sido en temas de autonomía y autogestión.

Pero estos acercamientos no siempre han sido positivos. Por ejemplo, la REI ha solicitado al Ministerio de Turismo cursos para guías mediante sendos oficios, sin resultados positivos; la ayuda de entidades públicas ha sido enfocada hacia las organizaciones de manufactura, pero de forma discontinua.

Por otro lado, se puede resaltar la visión de las mujeres del Comité de Turismo Manduriaco, del Rosal, de Mujeres y Medio Ambiente, y Turismo 


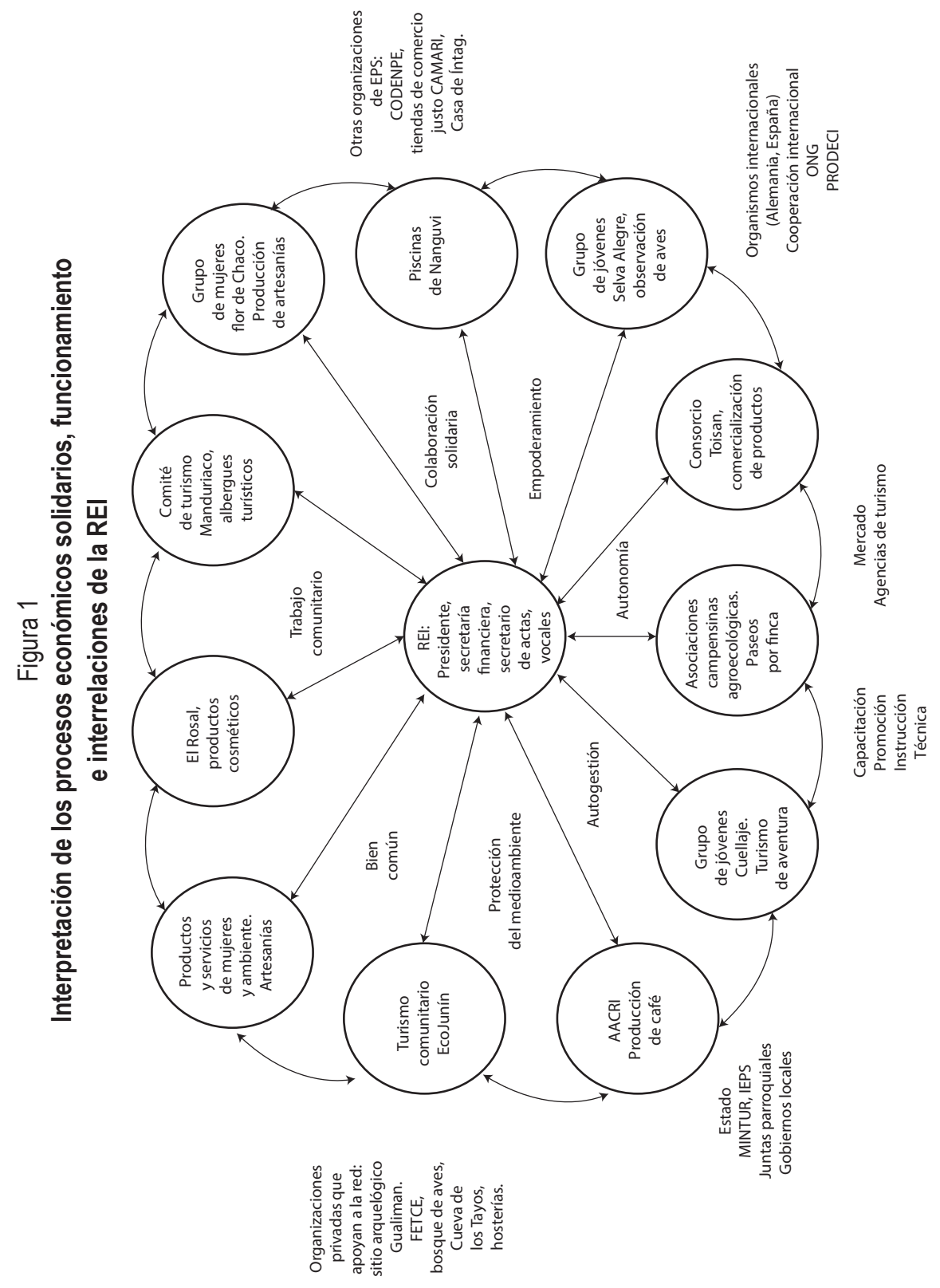

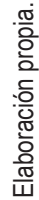


Eco Junín, ya que sienten que la parte social es la que mueve al sector, han logrado una participación en la red, envío de productos al exterior, e inclusive participación en la política, es decir, ha sido una forma ganar espacio, porque para las mujeres es más difícil conseguir un trabajo en la zona (J. A. 2016, entrevista personal).

Conjuntamente con el funcionamiento de una red de colaboración solidaria, se dan las fases del proceso económico solidario, como es la producción solidaria en donde todos los miembros de la red tienen acceso a los medios de producción, a capacitaciones para los servicios turísticos, producción en forma grupal y generación de trabajo para todos sus miembros, lo que permite el desarrollo de la vida humana más que del capital. Ellos también manejan una cooperación solidaria, donde la competencia está subordinada al principio de solidaridad entre sus miembros; su producción responsable con la naturaleza, usando en su mayoría tecnologías artesanales y con los consumidores -turistas- comprometidos con la preservación del medioambiente (Coraggio 2011, citado en Jácome 2014).

En cuanto a la comercialización justa y solidaria: existe una reducción de intermediarios, ya que son las mismas organizaciones solidarias las que tratan de comercializar los productos con los consumidores, turistas, asociaciones solidarias y empresas privadas en el exterior. Además, esta estrategia les permite mejorar los precios, fomentar la autonomía y el empoderamiento de las familias sobre lo que producen y los paquetes turísticos (Jácome 2014). El consumo responsable y solidario comienza por el autoconsumo de sus productos, y los que se generan por la actividad turística son los únicos que se comercializan solo para los externos; sin embargo, en todas sus actividades fomentan el bien vivir colectivo, favoreciendo a los trabajadores que producen, distribuyen y comercializan bienes y servicios y favorecen el mantenimiento del equilibrio dinámico de los ecosistemas. De esta forma, incentivan el consumo de los productos y servicios de la economía solidaria en lugar de productos de empresas que explotan a los trabajadores y degradan los ecosistemas (André 2013, citado en Jácome 2014).

Cabe resaltar que el dinero generado por los servicios comercializados por la red es distribuido en un $75 \%$ para la organización que provee el servicio o producto, y el $25 \%$ se utiliza para inversión propia de la red; todo excedente se reparte entre las organizaciones participantes. 
El sector de Íntag atraviesa por varias dificultades, especialmente por la amenaza de la degradación de su biodiversidad por actividades como la minería a cielo abierto y proyectos hidroeléctricos a gran escala, que coaptan las formas organizativas de las comunidades que pretenden mejorar sus condiciones, dentro de su lógica sostenible, tanto para los habitantes como para la naturaleza. A pesar de ello, la economía solidaria, a través de las organizaciones y el trabajo con la red, se manifiesta no solo para generar ganancias, sino principalmente para el fortalecimiento de sus lazos de retroalimentación política, social y económica, en principios y valores de su comunidad, como por ejemplo en mingas, organización de fiestas que engrandecen la parte cultural y en otras formas que no necesariamente deben ser económicas, pero que generan vínculos fuertes, que además respetan las heterogeneidades de las comunidades de cada parroquia, permitiendo así el desarrollo integral local.

Por otro lado, se puede observar que la idea de crear la REI fue en parte para producir y aprovechar los recursos naturales mediante una actividad turística comunitaria sostenible, que además sirve de nexo entre los miembros, amplía la riqueza del sector en beneficio de todos, sin dejar de lado que deben ser recíprocos con la comunidad por los recursos que se les permiten utilizar.

Igualmente en el Íntag, a través de la red de colaboración solidaria, han reorganizado los flujos económicos solidarios en territorio, aunque el desarrollo tecnológico es aún bajo, pero con gran apoyo humano que ayuda a propender un desarrollo económicamente viable, ecológicamente sostenible y socialmente justo para los miembros y de sus comunidades (Mance 2011).

En esta investigación cabe resaltar las voces de los actores internos y externos con varios de sus comentarios relevantes en diferentes aspectos, enfatizando el trabajo de la mujer, que antes era desvalorizado por solo dedicarse a las tareas del hogar y al cuidado de los niños, ahora les ha permitido romper paradigmas en su territorio y en lo posible manejar la equidad de género en sus actividades (S. R. 2016, entrevista personal).

El tener un nexo con la red y la actividad que se realiza genera una visión amplia en el manejo cooperativo, combinando una actividad económica con lo social. Así lo manifiesta un socio de la red: "Si no hubiera alianzas, tal vez no se lograría lo que hoy tenemos para seguir adelante, como organizaciones 
es más complicado avanzar solos". (V. L. 2016, entrevista personal). De esta forma, la REI ha sido fundamental en la zona entrelazando las diferentes áreas productivas, social, holístico y económico.

La filosofía principal que impulsa la red obliga a que los recursos que se generan en el territorio deben ser reinvertidos en el mismo, tal como indica Vaca (2016). Por ejemplo, la producción de café genera una dinámica económica en el territorio y a la vez aporta a la economía del país, creando plazas de trabajo, flujo económico y de esa forma reduce la migración de los jóvenes.

Existe una asociación de hosterías privadas que cuenta con ocho miembros de las parroquias Peñaherrera y García Moreno, que están registradas en el Ministerio de Turismo. Las organizaciones privadas en mención se dedican a una sola actividad que es el turismo; su propuesta es poner una oficina de información donde todos los miembros puedan ofrecer los servicios, y así establecer acuerdos (H. B. 2016, entrevista personal).

Se ha evidenciado una desconexión con la institución pública. En Íntag los recursos de la economía pública han dependido de la orientación de las políticas de Estado, como lo indica Coraggio (2012), y no se ha consultado a los habitantes con la debida importancia sobre las actividades que desarrollan en su territorio (S. N. 2016, entrevista personal).

Desde el GAD Municipal de Cotacachi y la Junta Parroquial se ha destinado un presupuesto para las organizaciones a través de proyectos, lo cual es favorable para apoyar en el desarrollo de las nuevas iniciativas de las organizaciones del cantón, incluyendo a Íntag.

\section{Discusiones finales}

El aporte y beneficio de las redes de colaboración solidaria es muy valioso en territorio, porque permite reducir el tiempo de circulación económica. Se producen más bienes que se pueden consumir dentro o fuera de la red, generando más excedentes y ampliando la riqueza para el bienestar de todos. Estas redes de colaboración promueven la conservación ambiental, desarrollan actividades autosostenibles locales, lo cual genera una diversificación de sus productos para atender la demanda del buen vivir de todos, y además crean fuertes vínculos sociales, culturales y económicos entre las organizaciones. 
El turismo es una alternativa viva para luchar a favor de la conservación del medioambiente como eje estratégico en la localidad, permite cobijar a otras actividades socioeconómicas como la agricultura, la manufactura de productos, los deportes, entre otras, haciendo posible una sostenibilidad económica y ambiental. Se puede precisar que la actividad turística ha generado vínculos con varios actores de la comunidad, alianzas solidarias y equidad de género.

Es importante entender que este tipo de organizaciones son heterogéneas, que se deben respetar y alimentar con estrategias basadas en la gestión propia, ya que sus realidades en territorio son muy diferentes de la urbanidad e incluso de otros lugares rurales, por lo cual además es indispensable un acompañamiento de la parte pública in situ. Como se explicó anteriormente, no hay una ayuda clara del sector público en proporcionar recursos económicos a través de proyectos de la red. Tampoco existe un acompañamiento del Estado, el cual no ha entendido que el fin no es solo comercializar productos, sino también luchar por el medioambiente y fortalecer al turismo por medio de sus organizaciones sociales; que al contrario, se las sigue mirando como microempresa, no como organizaciones con vínculos solidarios.

Adicionalmente, para fomentar el desarrollo de las redes en el marco de la economía solidaria y considerar el desarrollo de género y de territorio, como lo indica Jubeto, Guridi y Fernández-Villa (2014), se debe involucrar con su propuesta a más organizaciones de Íntag que promuevan circuitos más dinámicos. Se debe contar con encadenamientos además del conocimiento de una economía alternativa que permita la asignación de mayores recursos económicos a estas zonas, que por ahora no son suficientes en el cantón.

Es preciso señalar que en esta investigación no se ha tratado de medir exclusivamente la eficiencia de la REI por su rentabilidad económica, pues se restringiría únicamente a la evaluación de un proyecto capitalista que subsume a las otras esferas, lo que debe implicar el uso de los instrumentos sociales existentes y crear otros para evaluar el resultado de estas experiencias que involucren la esfera cultural, ambiental, política, económica y social, que son las que en realidad permiten resaltar todas estas formas de solidaridad, que se ha venido mencionando a lo largo de la investigación

Por otro lado, el medioambiente debe integrarse permanentemente a las actividades de la red, pero siempre respetando las cadenas y procesos natu- 
rales del ecosistema local, por lo que es necesario ampliar ese conocimiento en todo Íntag con una propuesta sostenible, como una mejor alternativa a las actividades extractivistas que se imponen desde fuera.

Además, es importante integrar a flujos culturales, ya que estas son un factor muy fuerte de unión, que se basa en un ideal para compartir conocimientos, comunicación, lenguajes, tradiciones, así como la retroalimentación de patrones éticos y morales, que ayuden a difundir el poder que tienen las comunidades en su dinámica propia. De esta forma, se recomienda establecer más acuerdos entre los actores que no integran la red, así como generar acuerdos con entidades del sector público.

Así mismo, son de suma importancia las alianzas con los sectores privados, públicos y otras organizaciones de la economía popular y solidaria para promocionar los productos turísticos de Íntag. Esto permitirá visibilizar con mayor facilidad tanto la experiencia de la red como los productos y servicios de las organizaciones que la integran.

Es necesario potenciar más el turismo comunitario en la zona, creando productos turísticos más estructurados en circuitos, que les permitirían atraer mayor demanda al sector, pero garantizando sustentablemente el buen vivir de las comunidades de Íntag y de la naturaleza. En este mismo punto, es importante hacer un mapeo de todas las organizaciones sociales y privadas que realizan una actividad turística en Íntag, con el fin de identificar el tipo de turismo al que se dedican, para proponer políticas desde la visión comunitaria, con enfoque en el turismo solidario comprometido con el medioambiente, científico y cultural.

Por otro lado, es indispensable constituir arreglos socioeconómicos solidarios más fuertes en base al turismo en la zona, para de esta forma incentivar el desarrollo endógeno sustentable de la red y exógeno en los ámbitos económico, social, político, cultural y ecológico, con un alto grado de credibilidad en esta propuesta, que a su vez atraiga el interés por ser parte de la red a otras organizaciones sociales del sector.

Adicionalmente, se debe tener presente que las experiencias comunitarias de la red deben ligarse de alguna forma con las personas que proveen los servicios privados y llegar a acuerdos de beneficios mutuos, para que todos los habitantes de Íntag se sientan involucrados en esta nueva alternativa de desarrollo. Es importante que sus proyectos respeten la lógica solidaria y 
el medioambiente, ya que es una forma óptima para lograr que el valle de Íntag sea conocido a nivel local y nacional. De esta forma, crear centros de información que promuevan las actividades turísticas de la zona a través de la red, con el apoyo de los privados, servirá para fortalecer a todo el sector.

Asimismo, para construir nuevas redes es necesario entender la realidad territorial con la teoría de redes de colaboración solidaria, que fomentan esta sinergia colectiva en otra lógica y que han articulado a los actores de la economía popular y solidaria en Ecuador. En ese sentido, la mayoría de estas organizaciones, si se encaminan a futuro, generarán adecuados circuitos económicos solidarios, con nuevos emprendimientos, los cuales se podrán articular con sus actividades, insistiendo en la coproducción y construcción conjunta de políticas públicas con los actores en temas de turismo y redes de desarrollo de territorio, aplicando otros indicadores que pasen de la esfera económica e involucren las esferas social, ambiental y política.

\section{Referencias}

Caracciolo Basco, Mercedes, y María del Pilar Foti Laxalde. 2003. Economía solidaria y el capital social. Buenos Aires: Paidós.

Coraggio, José Luis. 2009. Hacia un registro de actores (organizaciones socioeconómicas) y efectores de economía popular solidaria. Buenos Aires: Paidós SAICF.

---.2011. Economía social y solidaria: el trabajo antes que el capital. Quito: Abya-Yala / Fundación Rosa Luxemburgo.

---. 2012. "Hacia otra economía: teoría y práctica de la economía social y solidaria en América Latina". En Las tres corrientes vigentes de pensamiento y acción dentro del campo de la economía social y solidaria, editado por José Coraggio, 11-24. Buenos Aires: Paidós.

---.2013. Fundamentos de economía social y solidaria. Quito: Instituto de Altos Estudios Nacionales.

---, María Inés Arancibia y María Victoria Deux. 2010a. "Conceptos básicos”. En Guía para el mapeo y relevamiento de la economía popular solidaria en Latinoamérica y el Caribe, editado por José Luis Coraggio, María Inés Arancibia y María Victoria Deux, 16-17. Lima: Nova Print.

---. 2010b. Guía para el mapeo y relevamiento de la economía popular y solidaria en Latinoamérica y el Caribe. Lima: Nova Print.

EC CODELCO. 2016. "Dragones en el Edén”. CODELCO fuera de Íntag. Consulta: mayo de 2018. 〈http://codelcoecuador.com/acerca-de-Íntag/〉. 
EC Instituto Nacional de Estadística y Censos (INEC). 2010. “Censo de población y vivienda”. INEC. Consulta: abril de 2018. 〈https://bit.ly/2kkTh8e〉.

Gallicchio, Enrique. 2004. El desarrollo económico local en América Latina. ¿Estrategia económica o de construcción de capital social? Barcelona: CLAEH.

Guridi, Luis. 2014. "Presentación”. En diálogos de economía social y solidaria en Ecuador encuentros y desencuentros con las propuestas para otra economía, editado por Yolanda Jubeto, Luis Guridi y Maite Fernández-Villa, 13. Bilbao: Hegoa.

---, y Yolanda Jubeto. 2014. "Fundamentos conceptuales y principios de economía social y solidaria". En diálogos de economía social y solidaria en Ecuador, encuentros y desencuentros con las propuestas para otra economía, editado por Luis Guridi, Yolanda Jubeto, Maite Fernández-Villa, 45. Bilbao: Hegoa.

Instituto Interamericano de Cooperación para la Agricultura (IICA). 2013. Desarrollo territorial en el Íntag. Consulta: mayo de 2016. 〈https://bit.ly/2MMx4d6〉.

Jácome, Víctor. 2014. Introducción a la economía popular y solidaria. Quito: Instituto de Altos Estudios Nacionales.

Jubeto, Yolanda, Luis Guridi y Maite Fernández-Villa. 2014. Diálogos sobre la economía social y solidaria en el Ecuador. Bilbao: Lankopi, S. A.

Laville, Jean-Louis, y Luiz Inacio Gaiger. 2013. La economía solidaria y el capitalismo en la perspectiva de las transiciones. Bilbao: Lankopi S.A.

Mance, Euclides. 2001. La Revolución de las redes. Petrópolis: Vozes.

---. 2002. "Redes de colaboración solidaria". Socioeco org. Consulta: abril de 2015. «https:// bit.ly/2BkWqd1>.

---. 2010. Organizzare reti solidali. Strategie e strumenti per un altro sviluppo. Roma: EdUP.

---. 2011. Organización de redes solidarias: estrategias y herramientas para otro desarrollo. Petrópolis: Vozes.

---. 2013. "Redes de colaboración solidaria”. En Diccionario de la otra economía, editado por Patrick Viveret, Antonio David Cattani, José Luis Coraggio y Jean-Louis Laville, 297. Buenos Aires: Universidad Nacional de General Sarmiento.

Ortiz, Doris, y Patricio Mena. 2005. Páramo y contaminación. Quito: FLACSO Ecuador.

Red Ecoturística de Íntag (REI). 2011. "Alternativa de desarrollo en armonía con el medio ambiente”. Periódico Íntag. Consulta: abril de 2015. 〈https://bit.ly/32uSIJx〉.

Roux, Fanny. 2013. Turismo comunitario ecuatoriano, conservación ambiental y defensa de los territorios. Quito: FEPTCE. 